\title{
Implications of pre-transplant sarcopenia and frailty in patients with non-alcoholic steatohepatitis and alcoholic liver disease
}

\author{
Joseph S. Redman ${ }^{1}$, Matt Kaspar ${ }^{1}$, Puneet Puri ${ }^{1,2}$ \\ ${ }^{1}$ Division of Gastroenterology, Hepatology and Nutrition, West Hospital, Virginia Commonwealth University, Richmond, VA, USA; ${ }^{2}$ Division of \\ Gastroenterology, Hepatology and Nutrition, Hunter Holmes McGuire VA Medical Center, Richmond, VA, USA \\ Contributions: (I) Conception and design: All authors; (II) Administrative support: P Puri; (III) Provision of study materials or patients: None; (IV) \\ Collection and assembly of data: None; (V) Data analysis and interpretation: None; (VI) Manuscript writing: All authors; (VII) Final approval of \\ manuscript: All authors. \\ Correspondence to: Puneet Puri. Division of Gastroenterology, Hepatology and Nutrition, Hunter Holmes McGuire VA Medical Center, 1201 Broad \\ Rock Blvd, Richmond, VA 23249, USA. Email: puneet.puri@vcuhealth.org.
}

\begin{abstract}
Frailty manifesting as sarcopenia is an independent risk factor for mortality in cirrhosis, and often presents in low model for end-stage liver disease (MELD) patients. Its etiology is multifactorial, but key physiologic changes culminate in altered energy utilization in the fasting state, preferentially utilizing muscle amino acids for gluconeogenesis thereby promoting sarcopenia. Hyperammonemia alters the circulating amino acid profile, diminishing pro-muscle branched-chain amino acids like leucine. The metabolic syndrome worsens sarcopenia through multi-tissue insulin resistance. Alcohol also exacerbates sarcopenia as a direct muscle toxin and inhibitor of growth signaling. Therapy is aimed at alcohol cessation, frequent highprotein meals, branched-chain amino acid supplementation, and diminished time spent fasting. Moderate exercise can improve muscle mass and muscle quality, though precise exercise regimens have not yet been explicitly determined. Studies are ongoing into the effects of myostatin antagonists and insulin sensitizers. The Liver Frailty Index can predict patients most at risk of poor outcome and should be considered in the management of all cirrhotic patients. Specialty testing like dual-energy X-ray absorptiometry (DEXA) scanning and cross-sectional estimates of muscle mass are areas of active research and may play a future role in clinical risk-stratification.
\end{abstract}

Keywords: Sarcopenia; frailty; non-alcoholic steatohepatitis (NASH); alcohol; transplant

Received: 24 December 2019; Accepted: 06 July 2020; Published: 25 July 2022.

doi: $10.21037 /$ tgh-20-236

View this article at: http://dx.doi.org/10.21037/tgh-20-236

\section{Introduction}

When the model for end-stage liver disease (MELD) was implemented for liver allocation in 2002 it was praised for its objectivity and capacity for reducing liver transplant wait-list mortality (1). Unfortunately, significant pretransplant wait-list mortality persists, with growing interest to identify additional risk factors that might predict early mortality, particularly factors amenable to intervention. Already there are several known predictive characteristics not captured by the MELD-Na score, including age, metabolic co-morbidities, and frailty with its associated deficits in muscle mass and nutritional status. While age was originally included in the MELD calculation for predicting transjugular intrahepatic portosystemic shunt (TIPS)related mortality, this factor was removed from the MELD when applied to transplant allocation to avoid age-related bias. Nevertheless, frailty, while typically age-associated, represents an age-independent functional risk factor in cirrhotic populations that may better represent patients' overall fitness for transplant and perioperative mortality risks.

The concept of frailty has been well established in the geriatric and cancer literature, and has been more recently applied to transplant populations including liver transplant 
candidates. Ongoing efforts have been made to simplify and objectively quantify frailty in order to equitably apply its predictive capacity towards pre-transplant management. Although the underlying pathophysiology is multifactorial, it is believed to result from an aggregate decline of numerous systems, including neuromuscular, inflammatory, skeletal and endocrine systems. Features of frailty include sarcopenia, decreased cardiopulmonary reserve, diminished functional status deficits, and overt disability. These features are exacerbated by malnutrition and uncontrolled medical comorbidities (particularly heart disease and diabetes).

\section{Evaluating for sarcopenia}

Sarcopenia can be characterized by measures of total body muscle, muscle function/strength, and muscle quality as determined by fat content and lipid hormone metabolism. Simple but imprecise assessments include anthropomorphic measurements of body mass index (BMI), waist circumference, and mid-arm circumference to estimates fat composition and muscle mass. More robust and prognostic measures have since been described to better estimate overall body composition, including bioelectric impedance analysis (BIA) and dual-energy X-ray absorptiometry (DEXA). BIA involves holding a handheld device that delivers a weak electric current throughout the body. Changes in body composition (lipid-rich fat $v$ s. water-rich muscle) alters impedance, which is detected as a delay between the two hand-held contacts of the device. In general, BIA has been considered to be too variable for clinical use, though newer devices may be more reliable. Still, the "gold standard" for body composition assessment is considered to be the DEXA scan. More commonly used to measure bone density, the same instrument can be used to measure lean soft tissue (muscle) and by extension estimate the fat fraction by subtracting lean tissue from total body mass.

Radiographic studies are continually evolving to better quantify the anatomical distributions of muscle, subcutaneous and visceral fat [subcutaneous adipose tissue (SAT) and visceral adipose tissue (VAT)]. First described in 1985, the skeletal muscle index (SMI) approximates total body muscle from a single cross-sectional image at the level of the L3 vertebral body (SMI, or L3-SMI) (2). The SMI (and other radiographic variables) can be adjusted for the patient's sex, age and vertebral level, available under the Reference Analytic Morphomic Population (RAMP) tool at http://www.med.umich.edu/surgery/morphomics/ramp.
In general, cut-off values for sarcopenia in patients with cirrhosis are defined as SMI $<50 \mathrm{~cm}^{2} / \mathrm{m}^{2}$ in males and $<39 \mathrm{~cm}^{2} / \mathrm{m}^{2}$ in female patients (3). While alternative (simpler) radiographic measures have been more recently proposed, including the psoas muscle index (PMI), it should be noted that PMI has been found to correlate poorly with SMI, with overall less sensitivity for detecting sarcopenia $(4,5)$.

\section{Frailty assessment}

Frailty (diminished physical function due in part to sarcopenia) is now a known predictor of wait-list mortality independent of the MELD-Na score in liver transplant candidates (and perhaps an even better predictor of mortality in sarcopenic patients with a MELD-Na score $<18)$. In a comparative study (1), cirrhotic patients listed for liver transplantation with a MELD-Na $>12$ were assessed with four frailty indices including the Fried Frailty Instrument (FFI, assesses 5 domains of functionality, frail if score $\geq 3$ ), Short Physical Performance Battery (SPPB, frail if <9), Activities of Daily Living (ADL), and Instrumental ADL scales. Both the FFI and SPPB were found to be predictive of wait-list mortality, independent of the MELD$\mathrm{Na}$ score. For each 1-unit increase on the Fried Frailty assessment (increasing frailty) there was a MELD-adjusted $45 \%$ increased risk of wait-list mortality. Similarly, a 1-unit decrease in the SPPB (increasing frailty) was associated with a $19 \%$ MELD-adjusted increased risk in mortality.

It is notable that the FFI has both objective and subjective measures: hand-grip strength as quantified by a hand-held dynamometer, a 4-meter timed walk test, unintentional weight loss $<10$ pounds in the previous year, responses to a physical activity questionnaire over the last 4 weeks, and the patient's subjective assessment of personal exhaustion. Notwithstanding the valuable implications of patients' own subjective abilities to participate in their daily routines, the subcomponents of the test that most-predicted mortality were all objective: grip strength, unintentional weight loss, and physical inactivity. The SPPB, on the other hand, is entirely objective and requires no specific equipment, consisting of timed repeated chair stands, a sequence of balancing tests, and an 8 foot ( 2.44 meter) timed walk test. The advantages of either test are that they can be performed quickly, repeatedly, and in the clinical setting, negating the need for complex metabolic assessments (e.g., cardiopulmonary exercise testing, isokinetic muscle testing). Moreover, a hybrid test of the most objective and predictive components of both tests (age, 
3 grip strengths, 5 chair stands, 3 -second holding balance) can now be readily calculated as the Liver Frailty Index (LFI) from the authors' website: https://liverfrailtyindex.ucsf. edu. This assessment has since been subsequently validated as predictive for frailty in both cirrhotic and noncirrhotic populations (6).

\section{Pre-transplant outcomes}

Sarcopenia and frailty both predict morbidity and mortality in patients with cirrhosis. A prospective study demonstrated a significant increase in both overt and covert hepatic encephalopathy in hospitalized patients with sarcopenia (decreased mid-arm muscle circumference and handgrip strength), but not BMI (7). Another study utilized anthropometric measurements to estimate malnutrition and determined that both severe muscle mass depletion (midarm circumference $<5^{\text {th }}$ percentile) and severe fat depletion (triceps skinfold thickness $<5^{\text {th }}$ percentile) were independent predictors of mortality in patients awaiting transplant (8). Similarly, estimates of total body muscle using L3-SMI found that radiographic sarcopenia was an independent predictor of pre-transplant mortality (9). Hamaguchi et al. further assessed muscle quality by measuring intramuscular adipose content (IMAC) by CT scan at L3. With this measurement, they found additive post-transplant mortality risks for both pre-transplant SMI muscle mass and muscle quality (myosteatosis) (10). For patients waiting on the liver transplant list, sarcopenia progresses with an average loss in SMI of $1.3 \%$ per 100 days along with worsening myosteatosis as detected by $-2.5 \%$ Hounsfield units per 100 days, while visceral fat appears stable (11).

Of particular interest, both Lai et al. and Tandon et al. observed that the greatest sarcopenia-related mortality risk was in patients with MELD-Na scores less than 15 , suggesting that sarcopenia conveys a significant earlyonset mortality risk not effectively captured by the MELD$\mathrm{Na}$ score $(1,9)$. These findings echo earlier observations that sarcopenia correlates with mortality in patients with Child-Pugh A and Child-Pugh B, but not Child-Pugh C cirrhosis (12). Alameri et al. also reported the same nonlinear relationship between Child-Pugh status in chronic viral hepatitis patients with diminishing global function as measured by their six-minute walk distance $(6 \mathrm{MWD})$; specifically they noted a significant drop in functional status as patients progressed from chronic liver disease to ChildPugh A cirrhosis, with a smaller decrease towards ChildPugh B cirrhosis, but then found no further significant difference in the functional status of Child-Pugh B and C patients (13). The 6MWD was later shown to be predictive of mortality in patients listed for liver transplant (14).

\section{Post-transplant outcomes}

Several recent studies have looked at post-transplant outcomes of frail and sarcopenic patients. Pravisani et al. found that in a population with $35.1 \%$ sarcopenia in preliving donor transplant patients, $28.9 \%$ still had sarcopenia by L3-SMI at 1-year post-transplant (15). Similarly, Bhanji et al. found sarcopenia in $50 \%$ of 293 pre-transplant patients by L3-SMI, $90 \%$ of which maintained sarcopenia at 1 year, with an additional $16 \%$ new onset sarcopenia (11). Follow-up CT scans within the first year demonstrated further decrease in muscle mass (median loss $2.4 \mathrm{~cm}^{2} / \mathrm{m}^{2}$ ), worsening myosteatosis ( -5.0 Hounsfield units), and an increase in abdominal visceral fat $\left(4.9 \mathrm{~cm}^{2} / \mathrm{m}^{2}\right)$. From a functional perspective, Lai et al. recently determined that frailty worsens in the 3 months following liver transplant and improves modestly by 12 months, but with fewer than $40 \%$ achieving robustness (16). In a study of 69 posttransplant patients followed for a median of 2.8 years, there was ongoing evidence of sarcopenia in $45 \%$ by arm muscle area, with an even higher incidence of poor muscle function as measured by handgrip strength ( $71 \%$ with $<30 \mathrm{~kg})(17)$.

In the immediate post-operative period, a retrospective analysis demonstrated an increased risk of infections, longer ICU length of stay, and increased duration of hospitalization, but no apparently increased perioperative mortality risk (18). Longer-term outcomes, however, have been indeterminate. While one study suggests no additional risk of long-term mortality with sarcopenia (18), another notes a significant correlation between sarcopenia and long-term mortality after liver transplant (19). Among patients who underwent urgent inpatient evaluation and liver transplantation, sarcopenia predicted post-transplant mortality with higher rates of death at 1 year $(86 \% v s$. $95 \%)$ and 3 years ( $73 \%$ vs. $95 \%)$ compared to their nonsarcopenic peers (20).

Using CT estimates of muscle mass, DiMartini et al. found that $62 \%$ of pre-transplant patients with a BMI $>25$ (including both overweight and obese patients) were simultaneously sarcopenic, while $80 \%$ of cirrhotic patients with a "normal" BMI of 18.5-25 were also sarcopenic (21). In a retrospective analysis of 48,226 patients who had undergone liver transplant between 2002 and 2013 (Organ Procurement and Transplantation Network database), 
Chang et al. reported best survival outcomes for patients with a BMI of 28-37, even after stratifying for MELD score, suggesting that patients listed for transplant may do better if they are able to maintain some of their BMI weight in the pre-transplant period (22). Moreover, they found that the relationship between BMI and mortality after transplant was "U"-shaped, with highest mortality risk at the extremes of BMI ( $<19$ and $>40)$, with a nadir (least mortality) at a BMI of 34. For patients with MELD scores below 19, a low BMI $(<19)$ conferred additional mortality and graft loss risks compared to normal BMI transplant recipients (23). Post-transplant mortality outcomes for class-II obese patients (BMI 35-40) are comparable to those with normal BMI (24), however centers currently vary on the workup and listing of patients with morbid class-III obesity (BMI $>40$ ), including ongoing determinations about the role and timing of bariatric interventions (25).

\section{Pathophysiology of sarcopenia in cirrhosis}

The pathophysiology of muscle wasting in end-stage liver disease is multifactorial with significantly redundant crosstalk, but several key pathways predispose cirrhotic patients to a hyperactive catabolic state resulting in sarcopenia and subsequent frailty. Knowledge of the pathophysiology is important when identifying potential therapeutic targets for intervention.

First, the decompensated features of cirrhosis foment early satiety with subsequent reduced oral intake, nausea, and reduced physical activity. Patients later develop problems of malabsorption, reduced glycogen storage, increased systemic inflammation, and hypogonadism with decreased circulating testosterone (26). Hepatocellular dysfunction and portocaval shunting lead to hyperammonemia and a subsequent circulating amino acid imbalance. In particular, branched-chain amino acids (BCAA) are reduced, including the essential amino acid leucine, which are critical to maintaining and promoting skeletal muscle. Normally leucine, insulin, testosterone, and exercise-induced insulin-like growth factor (IGF-1) all converge to upregulate protein kinase B (Akt), which acts to inhibit FOXO-mediated protein degradation while simultaneously activating potent mTOR signaling for downstream muscle-protein synthesis. Growth hormone (GH) further contributes to IGF-1 signaling, however basal IGF-1 levels are significantly decreased in cirrhosis and demonstrate blunted response to exogenous $\mathrm{GH}$ administration (27). The collective result is diminished protein synthesis, increased proteolysis, and impaired satellite cell function. Satellite cells act as precursors to new muscle fibers as myogenically-committed multipotent stem cells located in the periphery of terminally differentiated muscle fibers. When activated by Akt, satellite cells proliferate and promote the growth and repair of muscle tissue.

With overall diminished Akt activity, skeletal muscle loss proceeds through two primary catabolic pathways, the ubiquitin-proteasome pathway (UPP) and the lysosomal/autophagy system. Proteolysis via UPP involves conjugation of damaged substrates with ubiquitin followed by degradation in the $26 \mathrm{~S}$ proteasome. Autophagy is a normal programmed cell death pathway that is activated in response to cell stress for the destruction and recycling of unnecessary, damaged, and/or dysfunctional cellular components. It involves the creation of a phagophore, followed by the capture of random and/or selected targets forming the autophagosome, fusion with the lysosome, and subsequent proteolysis of engulfed debris by lysosomal proteases (28).

As liver disease advances, skeletal muscle becomes increasingly important for ammonia detoxification, ultimately metabolizing up to $50 \%$ of circulating ammonia (29). Elevated ammonia depletes branched chain amino acids and potently activates skeletal muscle autophagy, exacerbating skeletal muscle wasting. Hyperammonemia further mediates expression of myostatin, a negativeregulator myokine which has been shown to circulate at high serum concentrations in cirrhotic patients (3). Myostatin activity is normally suppressed by IGF-1 signaling, but with blunted IGF-1 activity and ammoniamediated NF- $\kappa \mathrm{B}$ transcription, myostatin expression increases markedly. Myostatin becomes a potent inhibitor of skeletal muscle growth, diminishing protein synthesis through Akt/mTOR signaling and preventing satellite cell differentiation by promoting Smad3 interference of PCNA and Myo-D (30,31).

The fasting state is of particular concern in cirrhosis. Patients with liver cirrhosis have diminished glycogen stores, accelerated glycogenolysis (with accompanying hyperglycemia and glucotoxicity), and exhibit early-onset gluconeogenesis after short-term fasting, which predisposes to increased protein catabolic requirements and muscle depletion (32). Studies of the respiratory quotient (ratio of oxygen consumed to carbon dioxide produced) indicate an 
increased reliance on gluconeogenesis rather than fatty acids during the fasting state in cirrhosis, driving consumption of muscle-derived amino acids (33).

\section{Pathophysiology of sarcopenia in non-alcoholic fatty liver disease (NAFLD)}

In NAFLD and non-alcoholic steatohepatitis (NASH), metabolic dysregulation manifests as insulin resistance (IR), enhanced lipolysis and hypoalbuminemia resulting in an increase in free fatty acids (FFA), low adiponectin, and high myostatin levels. Excessive growth of adipose tissue recruits tissue macrophages that promote local inflammation, culminating in adipose tissue IR (34). There is a subsequent reduction in adiponectin and PPAR- $\gamma$, resulting in increased circulating FFA (inadequately sequestered due to diminished albumin levels). Excess FFAs are taken up by liver and muscle cells, promoting steatosis in both organs and exacerbating IR in those tissues. Skeletal muscle IR diminishes normal lipid metabolism by PPAR- $\delta$ and AMP-activated protein-kinase. Similarly, liver IR reduces PPAR- $\alpha$ and Nrf-2 while promoting SREBP-1c, DGAT-2, and the release of pro-inflammatory cytokines (35). This culminates in increased circulating insulin and glucagon, increased lipolysis, and further elevation of free circulating toxic FFA. Despite excessive triglyceride stores, diminished liver PPAR- $\alpha$ function reduces fatty acid betaoxidation. Excessive caloric intake further promotes denovo lipogenesis with simultaneous reduction in very lowdensity lipoprotein (VLDL) secretion, resulting in retained triglyceride and hepatic steatosis.

Obesity and sarcopenia are interrelated features of the metabolic syndrome. Indeed, muscle loss has been linked to visceral adiposity, which in turn has been shown to be a better predictor of metabolic complications, poor cardiovascular outcomes, and pre-transplant mortality than simple BMI (36). SAT and VAT are metabolically distinct, with the latter associated with more severe clinical morbidity and increased cardiovascular mortality (37). The anomaly of abundant fat stores with simultaneous sarcopenia complicates assumptions of malnutrition and limits the utility of simple anthropomorphic measurements like BMI in assessing frailty. As a result, research increasingly focuses on skeletal muscle mass as a surrogate for whole-body protein adequacy, giving rise to the concept of sarcopenic obesity (38). Cirrhotic patients with sarcopenic obesity have worse clinical outcomes and survival (39).

While there are no readily available assessments of insulin sensitivity, IR can be estimated by calculating Homeostasis Model Assessment index for insulin resistance (HOMA-IR), which is the normalized product of both circulating insulin and glucose levels. However, a response to glucose challenge (e.g., by a euglycemic hyperinsulinemic clamp) is thought to be more diagnostic of IR and elucidate a more time-dependent metabolic profile. A particular benefit of this technique is that the insulin dose can be adjusted to determine IR in the liver (low-insulin state) as compared to IR in the peripheral skeletal muscle (highinsulin state) (40).

\section{Pathophysiology of sarcopenia in alcoholic liver disease}

Alcohol use disorder has been steadily rising to become the third leading cause of preventable death in the United States (41). Interestingly, a direct comparison of cirrhotic patients with varying etiologies of liver disease suggests that alcohol-related liver disease produces a higher prevalence and greater severity of sarcopenia (33). While much of alcohol-related sarcopenia is due to the pathophysiology of cirrhosis as described above, alcohol also produces direct toxic effects in muscle. Exposing animal models to ethanol has been shown to directly impair protein synthesis (42). Alcohol inhibits mTOR signaling and protein synthesis, likely through upstream Akt inhibition and AMPK inactivation by dephosphorylation (43-45). Chronic alcohol exposure also produces a pro-inflammatory state, impaired muscle mitochondrial function, and subsequent oxidative stress $(46,47)$. While the liver metabolizes the majority of alcohol, there is evidence that skeletal muscle contributes significantly. However, excessive levels of ethanol and its toxic metabolite acetaldehyde can induce muscle loss through autophagy and, in extreme circumstances, induce rhabdomyolysis (48).

Patients presenting with alcoholic hepatitis are severely malnourished, and aggressive nutritional supplementation is required to improve survival outcomes (49). Alcoholic hepatitis often affects relatively younger patients (47.6 10.4 years), yet despite their younger age many already show radiographic evidence of sarcopenia that independently predicts longer hospital stay, sepsis, multiorgan dysfunction, hepatic encephalopathy, and higher MELD scores despite a diminished creatinine $(50,51)$. Patients with alcohol-related cirrhosis are also less likely to recover their muscle mass (L3-SMI) following liver transplantation. While obesity and diabetes were both associated with less sarcopenia at 1 year 
Table 1 Therapeutic strategies for sarcopenia in cirrhosis, with disease-specific interventions

\begin{tabular}{|c|c|}
\hline Alcohol cessation & Indicated in cirrhosis of all etiologies; alcohol exacerbates sarcopenia \\
\hline Daily requirements & $\begin{array}{l}\text { Recommend } 35-40 \mathrm{kcal} / \mathrm{kg} / \text { day, including protein intake } 1.2-1.5 \mathrm{~g} / \mathrm{kg} / \text { day (approximately } 20-25 \mathrm{~g} \text { of } \\
\text { high-quality protein with each meal) }\end{array}$ \\
\hline 6 oz. chicken & $36 \mathrm{~g}$ protein, $6.6 \mathrm{~g}$ BCAA \\
\hline 1 egg & $6.3 \mathrm{~g}$ of protein, $1.3 \mathrm{~g}$ of BCAA \\
\hline Late night snacks & Ample evidence for late-night snacks, reducing time spent fasting \\
\hline Leucine-rich supplementation & $12-14$ g/day (e.g., 5-6 servings of commercially available supplements) \\
\hline TIPS & May be considered in low MELD patients who already have indications \\
\hline Gut microbiome & Investigational status at present, ongoing research underway \\
\hline \multicolumn{2}{|l|}{ Diminished roles } \\
\hline Ammonia-directed therapies & $\begin{array}{l}\text { Hyperammonemia exacerbates sarcopenia, but anti-ammonia therapies beyond standard of care do } \\
\text { not improve sarcopenia }\end{array}$ \\
\hline Statins & $\begin{array}{l}\text { Statin therapies have pleotropic benefits in cirrhosis and NASH, but do not appear to have a role in } \\
\text { modifying sarcopenia }\end{array}$ \\
\hline Testosterone & Studies have shown limited benefit \\
\hline
\end{tabular}

BCAA, branched-chain amino acids; TIPS, transjugular intrahepatic portosystemic shunt; MELD, model for end-stage liver disease; NAFLD, non-alcoholic fatty liver disease; $\mathrm{NASH}$, non-alcoholic steatohepatitis.

post-transplant [odds ratio (OR) of 0.847 and 0.408 , respectively], patients transplanted for alcohol were more likely to have persistent sarcopenia (OR of 1.174) (15).

\section{Treatment-nutrition}

Treatment for frailty and sarcopenia begins with nutrition and exercise (Table 1). Nutrition efforts focus on highenergy high-protein diet (HEHP) and/or BCAA-rich protein supplementation, with increased meal frequency and late-evening snacks. European Society for Clinical Nutrition and Metabolism (ESPEN) guidelines on enteral nutrition in liver disease recommend overall energy intake of $35-40 \mathrm{kcal} / \mathrm{kg} / \mathrm{day}$, and protein intake $1.2-1.5 \mathrm{~g} / \mathrm{kg} / \mathrm{day}$ (approximately $20-25 \mathrm{~g}$ of high-quality protein with each meal) (52). This represents a $25-50 \%$ increase in recommended daily protein for normal adults $(0.8 \mathrm{~g} / \mathrm{kg} / \mathrm{d})$. Examples of high-quality protein (containing essential amino acids in a proportion needed by the human body) include $6 \mathrm{oz}$. of chicken ( $36 \mathrm{~g}$ protein, $6.6 \mathrm{~g} \mathrm{BCAA}), 6 \mathrm{oz}$. of salmon ( $34 \mathrm{~g}$ of protein, $5.9 \mathrm{~g} \mathrm{BCAA}), 6 \mathrm{oz}$. of peanuts (12 $\mathrm{g}$ of protein, $6.8 \mathrm{~g}$ of BCAA), or $1 \mathrm{egg}(6.3 \mathrm{~g}$ of protein, $1.3 \mathrm{~g}$ of BCAA). Adding two cans of Ensure Plus or Diabetic Resource to regular meals adds $710 \mathrm{kcal}$ per day. For hospitalized patients, nutritional needs and malnourishment risks can be assessed using the Royal Free Hospital Nutrition Prioritizing Tool (53), which accounts for tube feeding, fluid status, unintentional weight loss, and admission circumstances (i.e., alcoholic hepatitis) to determine how aggressive inpatient nutrition measures should be applied.

Multiple studies have also shown that late-night snacks 
(taken between 2,100 and $700 \mathrm{~h}$ ) offers better protection against muscle loss compared to calorie-equivalent daytime snacks, decreases lipid oxidation and improves nitrogen balance $(32,54)$. A HEHP diet of $1.56 \mathrm{~g} / \mathrm{kg} / \mathrm{d}$ in decompensated cirrhotic populations [Child-Turcotte-Pugh (CTP)-B/C] yields significant improvement in nitrogen balance over fasting individuals (55). Supplementation with leucine-rich protein (12-14 g/day) has also been of particular interest as a direct activator of mTOR-mediated protein synthesis. Cirrhotic patients placed on oral BCAA/ leucine supplementation show reversal of impaired mTOR signaling and autophagy measures (56).

\section{Treatment-exercise}

In geriatric patients and healthy controls, exercise increases skeletal muscle mass and functional capacity through an increase in insulin signaling, protein synthesis, AMPK activity, and satellite cell activation. However, the role of physical exercise regimens (either aerobic or resistance activity) in preventing or reversing sarcopenia in cirrhotic patients is unclear. Patients with cirrhosis experience reduced exercise capacity secondary to fatigue and complications secondary to portal hypertension, including ascites and hepatic encephalopathy. There is also increased muscle ammoniagenesis and hepatic ureagenesis in cirrhotics during exercise, potentially limiting traditional exercise benefits (57). While concerns have been published about how resistance activity, even at a moderate level, could predispose decompensated patients to transiently increased portal pressures and risk complications like variceal bleeding, a review of thirteen exercise regimens in cirrhotic patients found exercise training to be welltolerated, and results in improvement in exercise capacity and muscle mass (58). Accordingly, low-resistance aerobic exercise can be recommended to cirrhotic patients, e.g., walking 30-40 $\min 3$ to 4 times per week, and lifting light weights such as hand weights 2 to 3 times per week.

\section{Myostatin antagonists}

Myostatin antagonists have shown promise in reversing sarcopenia in murine models of cirrhosis, with efforts underway to describe their use in human clinical trials. In non-cirrhotic elderly patients, myostatin inhibitors significantly increased lean body mass and functional capacity (faster stair climbing, chair rising, and gait speed) (59). Monoclonal antibodies against the myostatin receptor activin IIb (e.g., bimagrumab) have also demonstrated efficacy in mice, with upcoming randomized controlled trials (60).

\section{Insulin sensitizers}

Insulin sensitizing therapy (e.g., metformin) has been proposed as a mechanism for reducing complications of NASH cirrhosis (61). Furthermore, post-transplant patients are prone to developing metabolic complications that reduce muscle mass and quality, including post-transplant diabetes mellitus (PTDM) and IR. Often referred to as "new-onset diabetes after transplant" (NODAT), this specific term may not be accurate since PTDM patients likely harbor early metabolic derangements making them predisposed to developing diabetes in the perioperative period. Indeed, pretransplant SAT on imaging has been found to accurately predict the risk of developing PTDM (62). Insulin sensitizers may reduce this complication.

\section{Gut microbiome}

The gut microbiome is known to contribute to energy balance and metabolism, and in some cases promote obesity (63). There is evidence now that the microbiome may also predispose to sarcopenia (64). Alcohol is known to change the microbiome and alter epithelial tight junctions, resulting in dysbiosis, pro-inflammatory cytokines and elevated lipopolysaccharides, which can predispose to sarcopenia $(33,65,66)$. Whether the microbiome can be altered to effect benefits in muscle maintenance is a focus of ongoing research.

\section{TIPS vs. liver transplant}

There are several reports that TIPS for refractory ascites may also reduce visceral fat and halt or reverse sarcopenia in patients with advanced liver disease $(67,68)$. Failure to improve sarcopenia after undergoing TIPS is associated with a significant increase in post-TIPS mortality $(9.8 \%$ vs. $43.5 \%$ at 40 months). In contrast, there is conflicting data regarding whether liver transplantation alters the course or prevalence of sarcopenia and frailty, as mentioned previously. Limited data are available regarding muscle function and quality post-transplant, however one retrospective study of 213 post-transplant patients demonstrated worsening LFI at 3 months, with only modest improvement at 12 months, suggesting that liver 
transplantation has limited impact on muscle function (16). It is unclear why TIPS might confer a significant benefit on the reversal of sarcopenia while liver transplantation may not. Mechanistically both therapies decrease portosystemic pressures, with transplant also improving hepatic synthetic function. Moreover, hyperammonemia following TIPS could be expected to worsen sarcopenia rather than improve it. Still, the explanation may be related to the use of posttransplant calcineurin inhibitors, which have been directly implicated as mediators of persistent post-transplant sarcopenia $(69,70)$.

\section{Other therapies}

Many other studied therapies have unclear, limited, or definitively no value. A careful evaluation of statin therapy in NAFLD has demonstrated no benefit in improving IR or liver fat percentage (40). Hyperammonemia, while known to contribute to sarcopenia, remains an unclear target for therapies; ammonia-reducing medicines have not yet been shown capable of reducing sarcopenia. Testosterone therapy has been considered but has also been demonstrated to have minimal benefit in cirrhotic patients, thought in part to be limited by high aromatase activity (71). Overall, the field of sarcopenia in cirrhosis is developing rapidly, with great interest in workup, evaluation, and the implementation of evidence-based interventions.

\section{Acknowledgments}

Funding: None.

\section{Footnote}

Provenance and Peer Review: This article was commissioned by the Guest Editors (Sanjaya K. Satapathy, David Bernstein, Nitzan Roth) for the series "Liver Transplantation in NASH and ALD" published in Translational Gastroenterology and Hepatology. The article has undergone external peer review.

Peer Review File: Available at https://tgh.amegroups.com/ article/view/10.21037/tgh-20-236/prf

Conflicts of Interest: All authors have completed the ICMJE uniform disclosure form (available at https://tgh. amegroups.com/article/view/10.21037/tgh-20-236/coif). The series "Liver Transplantation in NASH and ALD" was commissioned by the editorial office without any funding or sponsorship. The authors have no other conflicts of interest to declare.

Ethical Statement: The authors are accountable for all aspects of the work in ensuring that questions related to the accuracy or integrity of any part of the work are appropriately investigated and resolved.

Open Access Statement: This is an Open Access article distributed in accordance with the Creative Commons Attribution-NonCommercial-NoDerivs 4.0 International License (CC BY-NC-ND 4.0), which permits the noncommercial replication and distribution of the article with the strict proviso that no changes or edits are made and the original work is properly cited (including links to both the formal publication through the relevant DOI and the license). See: https://creativecommons.org/licenses/by-nc-nd/4.0/.

\section{References}

1. Lai JC, Feng S, Terrault NA, et al. Frailty predicts waitlist mortality in liver transplant candidates. Am J Transplant 2014;14:1870-9.

2. Shen W, Punyanitya $M$, Wang $Z$, et al. Total body skeletal muscle and adipose tissue volumes: estimation from a single abdominal cross-sectional image. J Appl Physiol (1985) 2004;97:2333-8.

3. Bhanji RA, Narayanan P, Allen AM, et al. Sarcopenia in hiding: The risk and consequence of underestimating muscle dysfunction in nonalcoholic steatohepatitis. Hepatology 2017;66:2055-65.

4. Ebadi M, Wang CW, Lai JC, et al. Poor performance of psoas muscle index for identification of patients with higher waitlist mortality risk in cirrhosis. J Cachexia Sarcopenia Muscle 2018;9:1053-62.

5. Alconchel F, Martínez-Alarcón L, Nicolás-López T, et al. Psoas Muscle Index Does Not Predict Post-Transplant Outcomes: A Series of 57 Liver Transplant Recipients. Transplant Proc 2020;52:549-52.

6. Wang CW, Lebsack A, Chau S, et al. The Range and Reproducibility of the Liver Frailty Index. Liver Transpl 2019;25:841-7.

7. Merli M, Giusto M, Lucidi C, et al. Muscle depletion increases the risk of overt and minimal hepatic encephalopathy: results of a prospective study. Metab Brain Dis 2013;28:281-4.

8. Alberino F, Gatta A, Amodio P, et al. Nutrition and survival 
in patients with liver cirrhosis. Nutrition 2001;17:445-50.

9. Tandon P, Ney M, Irwin I, et al. Severe muscle depletion in patients on the liver transplant wait list: its prevalence and independent prognostic value. Liver Transpl 2012;18:1209-16.

10. Hamaguchi Y, Kaido T, Okumura S, et al. Impact of Skeletal Muscle Mass Index, Intramuscular Adipose Tissue Content, and Visceral to Subcutaneous Adipose Tissue Area Ratio on Early Mortality of Living Donor Liver Transplantation. Transplantation 2017;101:565-74.

11. Bhanji RA, Takahashi N, Moynagh MR, et al. The evolution and impact of sarcopenia pre- and post-liver transplantation. Aliment Pharmacol Ther 2019;49:807-13.

12. Merli M, Riggio O, Dally L. Does malnutrition affect survival in cirrhosis? PINC (Policentrica Italiana Nutrizione Cirrosi). Hepatology 1996;23:1041-6.

13. Alameri HF, Sanai FM, Al Dukhayil M, et al. Six Minute Walk Test to assess functional capacity in chronic liver disease patients. World J Gastroenterol 2007;13:3996-4001.

14. Carey EJ, Steidley DE, Aqel BA, et al. Six-minute walk distance predicts mortality in liver transplant candidates. Liver Transpl 2010;16:1373-8.

15. Pravisani R, Soyama A, Isola M, et al. Chronological changes in skeletal muscle mass following living-donor liver transplantation: An analysis of the predictive factors for long-term post-transplant low muscularity. Clin Transplant 2019;33:e13495.

16. Lai JC, Segev DL, McCulloch CE, et al. Physical frailty after liver transplantation. Am J Transplant 2018;18:1986-94.

17. Alves BC, Bruch-Bertani JP, Galinatti CBM, et al. Obesity, dynapenia and high cardiovascular risk co-exist in postliver transplant setting: results of a cross-sectional study. Clin Res Hepatol Gastroenterol 2019;43:140-7.

18. Montano-Loza AJ, Meza-Junco J, Baracos VE, et al. Severe muscle depletion predicts postoperative length of stay but is not associated with survival after liver transplantation. Liver Transpl 2014;20:640-8.

19. Englesbe MJ, Patel SP, He K, et al. Sarcopenia and mortality after liver transplantation. J Am Coll Surg 2010;211:271-8.

20. Kuo SZ, Ahmad M, Dunn MA, et al. Sarcopenia Predicts Post-transplant Mortality in Acutely Ill Men Undergoing Urgent Evaluation and Liver Transplantation. Transplantation 2019;103:2312-7.

21. DiMartini A, Cruz RJ Jr, Dew MA, et al. Muscle mass predicts outcomes following liver transplantation. Liver Transpl 2013;19:1172-80.
22. Chang SH, Liu X, Carlsson NP, et al. Reexamining the Association of Body Mass Index With Overall Survival Outcomes After Liver Transplantation. Transplant Direct 2017;3:e172.

23. Bambha KM, Dodge JL, Gralla J, et al. Low, rather than high, body mass index confers increased risk for post-liver transplant death and graft loss: Risk modulated by model for end-stage liver disease. Liver Transpl 2015;21:1286-94.

24. Spengler EK, O'Leary JG, Te HS, et al. Liver Transplantation in the Obese Cirrhotic Patient. Transplantation 2017;101:2288-96.

25. Moctezuma-Velazquez C, Márquez-Guillén E, Torre A. Obesity in the Liver Transplant Setting. Nutrients 2019;11:2552.

26. Sinclair M, Gow PJ, Grossmann M, et al. Review article: sarcopenia in cirrhosis--aetiology, implications and potential therapeutic interventions. Aliment Pharmacol Ther 2016;43:765-77.

27. Assy N, Pruzansky Y, Gaitini D, et al. Growth hormonestimulated IGF-1 generation in cirrhosis reflects hepatocellular dysfunction. J Hepatol 2008;49:34-42.

28. Jiao J, Demontis F. Skeletal muscle autophagy and its role in sarcopenia and organismal aging. Curr Opin Pharmacol 2017;34:1-6.

29. Levitt DG, Levitt MD. A model of blood-ammonia homeostasis based on a quantitative analysis of nitrogen metabolism in the multiple organs involved in the production, catabolism, and excretion of ammonia in humans. Clin Exp Gastroenterol 2018;11:193-215.

30. Frost RA, Lang CH. Multifaceted role of insulin-like growth factors and mammalian target of rapamycin in skeletal muscle. Endocrinol Metab Clin North Am 2012;41:297-322, vi.

31. McDaniel J, Davuluri G, Hill EA, et al. Hyperammonemia results in reduced muscle function independent of muscle mass. Am J Physiol Gastrointest Liver Physiol 2016;310:G163-70.

32. Plank LD, Gane EJ, Peng S, et al. Nocturnal nutritional supplementation improves total body protein status of patients with liver cirrhosis: a randomized 12-month trial. Hepatology 2008;48:557-66.

33. Dasarathy J, McCullough AJ, Dasarathy S. Sarcopenia in Alcoholic Liver Disease: Clinical and Molecular Advances. Alcohol Clin Exp Res 2017;41:1419-31.

34. Suganami T, Ogawa Y. Adipose tissue macrophages: their role in adipose tissue remodeling. J Leukoc Biol 2010;88:33-9.

35. Jornayvaz FR, Samuel VT, Shulman GI. The role 
of muscle insulin resistance in the pathogenesis of atherogenic dyslipidemia and nonalcoholic fatty liver disease associated with the metabolic syndrome. Annu Rev Nutr 2010;30:273-90.

36. Linge J, Borga M, West J, et al. Body Composition Profiling in the UK Biobank Imaging Study. Obesity (Silver Spring) 2018;26:1785-95.

37. Davidson LE, Hunt SC, Adams TD. Fitness versus adiposity in cardiovascular disease risk. Eur J Clin Nutr 2019;73:225-30.

38. Stenholm S, Harris TB, Rantanen T, et al. Sarcopenic obesity: definition, cause and consequences. Curr Opin Clin Nutr Metab Care 2008;11:693-700.

39. Hara N, Iwasa M, Sugimoto R, et al. Sarcopenia and Sarcopenic Obesity Are Prognostic Factors for Overall Survival in Patients with Cirrhosis. Intern Med 2016;55:863-70.

40. Braun LR, Feldpausch MN, Czerwonka N, et al. Effects of Pitavastatin on Insulin Sensitivity and Liver Fat: A Randomized Clinical Trial. J Clin Endocrinol Metab 2018;103:4176-86.

41. QuickStats. Age-Adjusted Death Rates Attributable to Alcohol-Induced Causes, by Race/Ethnicity — United States, 1999-2015. MMWR Morb Mortal Wkly Rep 2017;66:491.

42. Preedy VR, Keating JW, Peters TJ. The acute effects of ethanol and acetaldehyde on rates of protein synthesis in type I and type II fibre-rich skeletal muscles of the rat. Alcohol Alcohol 1992;27:241-51.

43. Steiner JL, Lang CH. Alcohol impairs skeletal muscle protein synthesis and mTOR signaling in a timedependent manner following electrically stimulated muscle contraction. J Appl Physiol (1985) 2014;117:1170-9.

44. Laplante M, Sabatini DM. mTOR signaling in growth control and disease. Cell 2012;149:274-93.

45. Liangpunsakul S, Sozio MS, Shin E, et al. Inhibitory effect of ethanol on AMPK phosphorylation is mediated in part through elevated ceramide levels. Am J Physiol Gastrointest Liver Physiol 2010;298:G1004-12.

46. Liguori I, Russo G, Aran L, et al. Sarcopenia: assessment of disease burden and strategies to improve outcomes. Clin Interv Aging 2018;13:913-27.

47. Haida M, Yazaki K, Kurita D, et al. Mitochondrial dysfunction of human muscle in chronic alcoholism detected by using 31P-magnetic resonance spectroscopy and near-infrared light absorption. Alcohol Clin Exp Res 1998;22:108S-110S.

48. Thapaliya S, Runkana A, McMullen MR, et al. Alcohol- induced autophagy contributes to loss in skeletal muscle mass. Autophagy 2014;10:677-90.

49. Fung P, Pyrsopoulos N. Emerging concepts in alcoholic hepatitis. World J Hepatol 2017;9:567-85.

50. Crabb DW, Im GY, Szabo G, et al. Diagnosis and Treatment of Alcohol-Associated Liver Diseases: 2019 Practice Guidance From the American Association for the Study of Liver Diseases. Hepatology 2020;71:306-33.

51. Al-Azzawi Y, Albo B, Fasullo M, et al. Sarcopenia is associated with longer hospital stay and multiorgan dysfunction in alcoholic hepatitis. Eur J Gastroenterol Hepatol 2020;32:733-8.

52. Plauth M, Cabre E, Riggio O, et al. ESPEN Guidelines on Enteral Nutrition: Liver disease. Clin Nutr 2006;25:285-94.

53. Amodio P, Bemeur C, Butterworth R, et al. The nutritional management of hepatic encephalopathy in patients with cirrhosis: International Society for Hepatic Encephalopathy and Nitrogen Metabolism Consensus. Hepatology 2013;58:325-36.

54. Dasarathy S. Consilience in sarcopenia of cirrhosis. J Cachexia Sarcopenia Muscle 2012;3:225-37.

55. Dichi JB, Dichi I, Maio R, et al. Whole-body protein turnover in malnourished patients with child class B and C cirrhosis on diets low to high in protein energy. Nutrition 2001;17:239-42.

56. Tsien CD, McCullough AJ, Dasarathy S. Late evening snack: exploiting a period of anabolic opportunity in cirrhosis. J Gastroenterol Hepatol 2012;27:430-41.

57. Didsbury M, McGee RG, Tong A, et al. Exercise training in solid organ transplant recipients: a systematic review and meta-analysis. Transplantation 2013;95:679-87.

58. Jones JC, Coombes JS, Macdonald GA. Exercise capacity and muscle strength in patients with cirrhosis. Liver Transpl 2012;18:146-51.

59. Becker C, Lord SR, Studenski SA, et al. Myostatin antibody (LY2495655) in older weak fallers: a proofof-concept, randomised, phase 2 trial. Lancet Diabetes Endocrinol 2015;3:948-57.

60. Morvan F, Rondeau JM, Zou C, et al. Blockade of activin type II receptors with a dual anti-ActRIIA/IIB antibody is critical to promote maximal skeletal muscle hypertrophy. Proc Natl Acad Sci U S A 2017;114:12448-53.

61. Vilar-Gomez E, Vuppalanchi R, Desai A, et al. Long-term metformin use may improve clinical outcomes in diabetic patients with non-alcoholic steatohepatitis and bridging fibrosis or compensated cirrhosis. Aliment Pharmacol Ther 2019;50:317-28. 
62. Vaughn VM, Cron DC, Terjimanian MN, et al. Analytic morphomics identifies predictors of new-onset diabetes after liver transplantation. Clin Transplant 2015;29:458-64.

63. Vallianou N, Stratigou T, Christodoulatos GS, et al. Understanding the Role of the Gut Microbiome and Microbial Metabolites in Obesity and Obesity-Associated Metabolic Disorders: Current Evidence and Perspectives. Curr Obes Rep 2019;8:317-32.

64. Ticinesi A, Nouvenne A, Cerundolo N, et al. Gut Microbiota, Muscle Mass and Function in Aging: A Focus on Physical Frailty and Sarcopenia. Nutrients 2019;11:1633.

65. Bajaj JS. Alcohol, liver disease and the gut microbiota. Nat Rev Gastroenterol Hepatol 2019;16:235-46.

66. Dunagan M, Chaudhry K, Samak G, et al. Acetaldehyde disrupts tight junctions in Caco-2 cell monolayers by a protein phosphatase 2A-dependent mechanism. Am J Physiol Gastrointest Liver Physiol 2012;303:G1356-64.

doi: $10.21037 /$ tgh-20-236

Cite this article as: Redman JS, Kaspar M, Puri P. Implications of pre-transplant sarcopenia and frailty in patients with nonalcoholic steatohepatitis and alcoholic liver disease. Transl Gastroenterol Hepatol 2022;7:29.
67. Kohi MP, Lai JC. Impact of Muscle Gain after Transjugular Intrahepatic Portosystemic Shunt Creation on Patients Awaiting Liver Transplantation. J Vasc Interv Radiol 2019;30:874-5.

68. Tsien C, Shah SN, McCullough AJ, et al. Reversal of sarcopenia predicts survival after a transjugular intrahepatic portosystemic stent. Eur J Gastroenterol Hepatol 2013;25:85-93.

69. Sakuma K, Yamaguchi A. The functional role of calcineurin in hypertrophy, regeneration, and disorders of skeletal muscle. J Biomed Biotechnol 2010;2010:721219.

70. Sakuma K, Nakao R, Aoi W, et al. Cyclosporin A treatment upregulates Id 1 and Smad3 expression and delays skeletal muscle regeneration. Acta Neuropathol 2005;110:269-80.

71. Dasarathy S, Mullen KD, Dodig M, et al. Inhibition of aromatase improves nutritional status following portacaval anastomosis in male rats. J Hepatol 2006;45:214-20. 